\title{
Infection prevention and control in nursing severe coronavirus disease (COVID-19) patients during the pandemic
}

\author{
Lei Ye ${ }^{1}$, Shulan Yang ${ }^{2 *}$ and Caixia Liu²
}

Keywords: COVID-19, Infection prevention and control, Critically ill patients, Pandemic

The novel coronavirus disease 2019 (COVID-19) is now worldwide publicity. Five to $20 \%$ of the total COVID-19 positive cases required admission to an intensive care unit (ICU) and the mortality rate was approximately $50 \%$ among critically ill patients who developed acute respiratory distress syndrome [1-5]. Deeply concerned by the spread and severity, the World Health Organization (WHO) characterized COVID-19 as a pandemic in March 2020. In February, Wuhan was facing a sudden shortage of health workers induced by the COVID-19 pandemic. The Chinese health authorities reported that 3019 Chinese health workers were infected with COVID-19, of which 10 died [6]. Front-line health workers are at high risk of infection. Inadequate awareness and precautionary measures, patient overload, and staff burnout are considered as relevant reasons for health worker infections. As an emergency measure, the China government dispatched 189 national medical teams comprising more than twenty-thousand health workers from all over the country who volunteered to combat COVID-19 in Hubei. They had been working together with local health workers and successfully controlled the development of the epidemic. The goal of "Zero" COVID-19 infection among health workers was achieved. Actually, from February 12 to April 9, 9282 health worker COVID-19 cases were reported by the US Center for Disease Control and Prevention, and contacts

\footnotetext{
* Correspondence: 947373396@qq.com

${ }^{2}$ Department of Nursing, Zhejiang Hospital, 12 Lingyin Road, Hangzhou 310013, China

Full list of author information is available at the end of the article
}

with COVID-19 patients in health care, household, and community settings were all detected [7]. COVID-19 infections among health workers are common and fatal to the health system. Infection among health workers may cause widespread transmission within the system and even lead to the collapse of the whole services. And this was what exactly happened in Harbin in the past weeks; a persisting cluster centered on an 87-year-old inpatient infected more than eighty people, including 8 health workers. The affected hospital urgently suspended routine medical services as a result.

Based on Wuhan's experience, it is critical to develop tailored infection prevention and control (IPC) protocols for both workplace and non-occupational settings and to conduct effective IPC training. Thus, the following suggestions were summarized based on the first-hand experience of a national medical team from Zhejiang, to facilitate the development of IPC protocols in critical care settings.

\section{Appropriate implementing of PPE}

Generally, all health workers should implement appropriate personal protective equipment (PPE) regarding contact and droplet precautions based on recommendations by WHO [8]. For health workers in ICU, advanced protections are required during routine intensive care and airborne precautions are considered as airborne transmission may happen during aerosol-generating procedures. The implementation of PPE may be different by option in certain practices. In our experience, the most protective choices were made and the "zero" medical 
infection rate was treated as the top priority that all staff were equipped from head to toe. Compared to official recommendations, we selected some additional PPE during intensive care, such as an extra medical face mask outside the respirator, and both face shield and goggles (see Fig. 1). Additional PPE may increase the risk of sharp injuries and increase the difficulty of donning and doffing. To lower the incidence of adverse events, sequences of donning and doffing PPE were carefully developed based on the above selections through thorough group discussions and agreement was reached among the team.

\section{Donning and doffing PPE under the three-zone double-channel structure}

The ward was reconfigured into a three-zone doublechannel structure before accepting COVID-19 patients. In this design, the ward was divided into several working areas according to cleanliness and the moving lines of patients and medical staff were fixed (see Fig. 2). The patient care area was identified as contaminated, and all staff were fully equipped with PPE before entering the buffer area. When doing doffing, all staff took off the additional PPE during intensive care (such as the fluidresistant gown and face shield) in the first buffer area that was near the patient care area. In the second buffer area, staff doffed the coverall and goggles. Finally, in the clean area, all staff removed the remaining PPE and conducted personal hygiene. We also developed reasonable shift rotations determined by the most tolerable shift lengths to prolong the use of PPE. In a 4-6h shift, health care workers avoided eating, watering, and toileting.

\section{Real-time on-site IPC surveillance}

To strengthen IPC, an inspector was set to facilitate the routine IPC management by on-site monitoring. Basically, the inspector was responsible for supervising the adherence of donning and doffing procedures of each health worker and real-time surveillance. In this way, some highrisk intensive interventions were identified and improvement measures were implemented promptly. COVID-19specific precautions were drawn among the team consequently, such as waste management. According to recent reports, not only respiratory specimens but also serum, urine, and stool specimens might be positive for COVID19. Even though no further IPC advice was provided, advanced procedures for waste managing were necessary, such as collecting respiratory and non-respiratory wastes in covered containers filled with chlorinated disinfectants and discarding in fastened double-layered medical waste garbage bags.

\begin{tabular}{|c|c|c|c|c|}
\hline \multirow{12}{*}{ 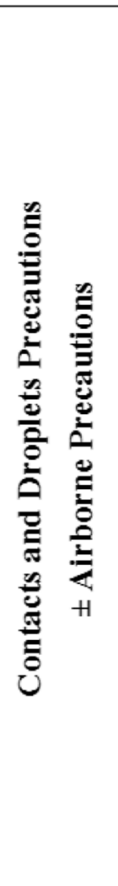 } & \multirow{4}{*}{\begin{tabular}{|c|} 
PPE \\
Masks \\
\end{tabular}} & Refers to & \multirow{3}{*}{$\begin{array}{c}\text { WHO } \\
\sqrt{ }\end{array}$} & \multirow{3}{*}{$\begin{array}{c}\begin{array}{c}\text { Wuhan } \\
\text { Experience }\end{array} \\
\sqrt{ } \sqrt{ } \\
\pm\end{array}$} \\
\hline & & Cap / Hair covers & & \\
\hline & & Medical face mask & & \\
\hline & & Respirator & \pm & $\sqrt{ }$ \\
\hline & \multicolumn{2}{|c|}{ Coverall } & $\begin{array}{l}\text { Not required during } \\
\text { routine care }\end{array}$ & $\sqrt{ }$ \\
\hline & \multicolumn{2}{|c|}{ Gown / Fluid-resistant Gown } & $\sqrt{ }$ & \\
\hline & \multicolumn{2}{|c|}{ Apron } & $\begin{array}{l}\text { Not required during } \\
\text { routine care }\end{array}$ & \pm \\
\hline & \multicolumn{2}{|c|}{ Gloves } & $\sqrt{ }$ & $\sqrt{ } \sqrt{ }$ \\
\hline & \multirow{2}{*}{ Eye protection } & Goggles & \multirow{2}{*}{$\sqrt{ }$} & $\sqrt{ }$ \\
\hline & & Face shield & & \pm \\
\hline & \multicolumn{2}{|c|}{ Shoes Covers / Boots } & $\begin{array}{l}\text { Not required during } \\
\text { routine care }\end{array}$ & $\sqrt{ }$ \\
\hline & \multicolumn{2}{|c|}{ Changing PPE when caring different patient } & $\sqrt{ }$ & $\sqrt{ }$ \\
\hline
\end{tabular}

Fig. 1 PPE selections for ICU setting 


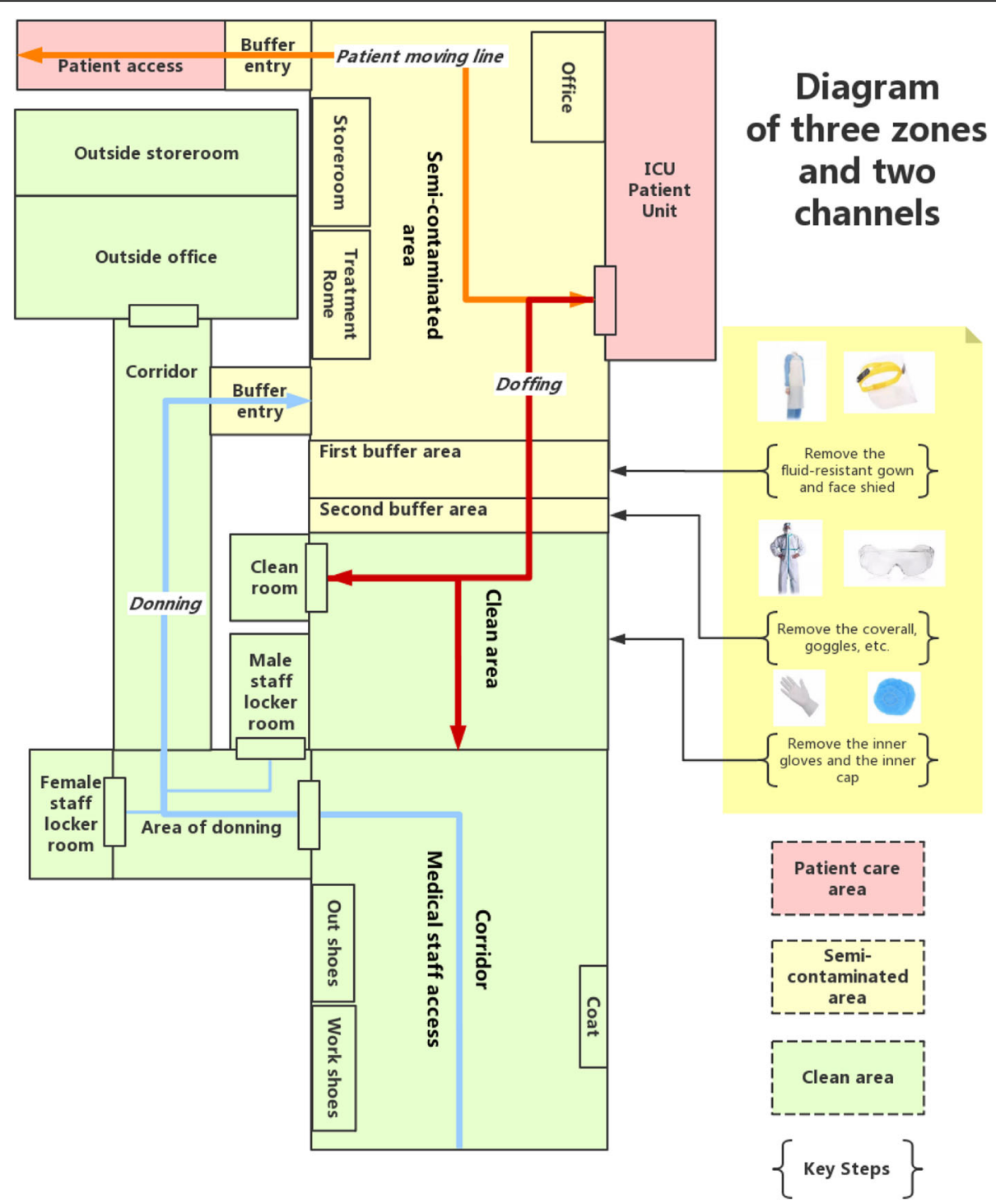

Fig. 2 Diagram of the three-zone two-channel structure

\section{IPC management in non-occupational settings}

Timely after arriving Wuhan, we established the ICP team and developed our own practical ICP procedures in non-occupational settings as well. We strictly ruled our behaviors during traffic routes and in the residential region and facilitated the whole team with a remote communication and collaboration platform using cellphone applications to strengthen communication. Same as what we do in the ward, we established the three-area double-channel structure and fixed our moving line. Besides, we developed behavior codes among the team, such as limiting gatherings and personnel contacts, routine disinfection of contact surfaces (handphone, doorknob, handle, etc.), and frequent hand hygiene on certain occasions.

\section{Conclusions}

All information provided in this paper is to strengthen the clinical practice in critical care settings and to better protect front-line health workers in nursing severe COVID-19 patients. The "zero" medical infection rate in our experience was hard won but worth fighting for.

\section{Acknowledgements}

Not applicable.

\section{Authors' contributions}

Shulan Yang and Lei Ye were the major contributors in writing and revising the manuscript. All authors read and approved the final manuscript.

Funding

Not applicable. 
Availability of data and materials

Data sharing not applicable to this article as no datasets were generated or analyzed during the current study.

\section{Ethics approval and consent to participate}

Not applicable.

\section{Consent for publication}

Not applicable.

\section{Competing interests}

The authors declare that they have no competing interests.

\section{Author details}

'Department of Critical Care Medicine, Zhejiang Hospital, 12 Lingyin Road, Hangzhou 310013, China. ${ }^{2}$ Department of Nursing, Zhejiang Hospital, 12 Lingyin Road, Hangzhou 310013, China.

Received: 2 June 2020 Accepted: 8 June 2020

Published online: 12 June 2020

\section{References}

1. Yang X, et al. Clinical course and outcomes of critically ill patients with SARS-CoV-2 pneumonia in Wuhan, China: a single-centered, retrospective, observational study. Lancet Respir Med. 2020;8(5):475-81.

2. Wang D, et al. Clinical characteristics of 138 hospitalized patients with 2019 novel coronavirus-infected pneumonia in Wuhan, China. JAMA. 2020; 323(11):1061.

3. Livingston E, Bucher K. Coronavirus Disease 2019 (COVID-19) in Italy. JAMA. 2020;323(14):1335. https://doi.org/10.1001/jama.2020.4344.

4. Di Lorenzo G, Di Trolio R. Coronavirus Disease (COVID-19) in Italy: Analysis of Risk Factors and Proposed Remedial Measures. Front Med. 2020;7:140. https://doi.org/10.3389/fmed.2020.00140.

5. Arentz M, Yim E, Klaff L, et al. Characteristics and Outcomes of 21 Critically III Patients With COVID-19 in Washington State. JAMA. 2020;323(16):1612-14. https://doi.org/10.1001/jama.2020.4326.

6. Xiang $Y$, et al. Tribute to health workers in China: a group of respectable population during the outbreak of the COVID-19. Int J Biol Sci. 2020;16(10): 1739-40.

7. Characteristics of health vare personnel with COVID-19 - United States, February 12-April 9, 2020. MMWR Morb Mortal Wkly Rep 2020;69:477-481. https://doi.org/10.15585/mmwr.mm6915e6.

8. Infection prevention and control during health care when COVID-19 is suspected: interim guidance, 19 March 2020. https:/www.who.int/ publications-detail/infectionprevention-and-control-during-healthwhennovel-coronavirus-(ncov)-infection-is-suspected20200125. (Accessed 19 May 2020).

\section{Publisher's Note}

Springer Nature remains neutral with regard to jurisdictional claims in published maps and institutional affiliations. 